\title{
La zone grise de l'allophonie : « mouvements secondaires » et scolarisation des «UPE2A italiens » de Strasbourg
}

The Grey Zone of Allophony: "Secondary Movements" and Schooling of the "Italians UPE2A" in Strasbourg

La zona gris de la alofonía: "movimientos secundarios» y escolarización de los «UPE2A italianos» de Estrasburgo

\section{Simona Tersigni et Lorenzo Navone}

\section{(2) OpenEdition}

\section{Journals}

Édition électronique

URL : https://journals.openedition.org/remi/11796

DOI : 10.4000/remi. 11796

ISSN : $1777-5418$

Éditeur

Université de Poitiers

Édition imprimée

Date de publication : 1 décembre 2018

Pagination : 119-142

ISBN : 979-10-90426-63-4

ISSN : 0765-0752

\section{Référence électronique}

Simona Tersigni et Lorenzo Navone, « La zone grise de l'allophonie : «mouvements secondaires » et scolarisation des «UPE2A italiens » de Strasbourg », Revue européenne des migrations internationales [En ligne], vol. 34 - n4 | 2018, mis en ligne le 01 janvier 2021, consulté le 15 avril 2022. URL : http:// journals.openedition.org/remi/11796; DOI : https://doi.org/10.4000/remi.11796 


\section{La zone grise de I'allophonie: " mouvements secondaires" et scolarisation des "UPE2A italiens " de Strasbourg}

\section{Simona Tersigni ${ }^{1}$ et Lorenzo Navone ${ }^{2}$}

Cet article propose une lecture de la sociogenèse propre aux recompositions catégorielles du système éducatif français au sujet d'une typologie spécifique d'élèves primo-arrivants. Leur situation conduit à relier la micro-échelle des dynamiques scolaires du dispositif UP2EA ${ }^{3}$ avec la reconfiguration de I'architecture des frontières internes et extérieures de l'espace européen. Une telle architecture se nourrit entre autres de politiques linguistiques dont il importe d'observer l'impact au sein des politiques éducatives nationales, en l'occurrence par la catégorisation scolaire d'élèves allophones depuis $2012^{4}$.

Pour ce faire, nous analyserons la situation des "UPE2A italiens de Strasbourg ", autrement dit des élèves scolarisés (et souvent nés) en Italie de parents ayant déjà migré du Maghreb, avec lesquels ils ont effectué une mobilité transalpine. Pour définir leur mobilité, nous employons le terme de " mouvement secondaire " qui a été initialement employé par le HCNUR pour désigner le contournement du règlement du Parlement européen et du Conseil européen ( ${ }^{\circ} 604-2013$ du 26/06/2013) dit Dublin III (Tazzioli et Garelli, 2018). Ces enfants et ces jeunes qui quittent I'Italie avec leur famille n'ont pas d'interdiction officielle de se déplacer dans d'autres pays membres de I'UE, contrairement aux cas des demandeurs d'asile. Cependant, en tant que "surplus scolaire " en provenance d'un autre pays européen, une fois les délais d'attente dépassés (jusqu'à neuf mois parfois), ils sont pris dans " une forme latente et plus modérée " d'allophonie, laquelle renvoie tout de même à une dynamique

1 Sociologue, Maître de conférences, SOPHIAPOL, Université de Paris Ouest Nanterre, 200 avenue de la République, 92001 Nanterre ; stersigni@ parisnanterre.fr

2 Docteur en sociologie (Université de Gênes, Italie), ATER à I'Université de Strasbourg, Chargé de recherche dans le cadre du programme Evascol, INSHEA, 58-60 avenue des Landes, 92150 Suresnes ; lo.navone@gmail.com

3 Unité pédagogique pour élèves allophones arrivants : il s'agit d'un dispositif scolaire appuyé sur une forme de ségrégation spatiale et pédagogique, censé être limité dans le temps (un an environ) et qui vise l'enseignement du français scolaire à des élèves ayant connu une migration internationale récente, avec une insertion progressive en classe ordinaire.

4 Cf. Circulaire $n^{\circ} 2012-141$ du 02/10/2012 organisant la scolarité des élèves allophones nouvellement arrivés (EANA). 
de segmentation sociale propre à l'univers scolaire (Armagnague-Roucher et Tersigni, 2019).

En tant que catégorie institutionnelle, l'allophonie renvoie à un esprit prétendument objectif reposant sur des idées qui sont communes, "non parce qu'elles seraient en fait partagées par beaucoup d'individus, mais parce qu'elles font autorité " (Descombes, 1996 : 291). Quelles sont ces idées imposant leur autorité dans notre situation ? II s'agit de la décision de cesser de nommer comme " non francophones ${ }^{5}$ des élèves primo-arrivants pour privilégier l'étiquette $d^{\prime}$ " allophones ". Celle-ci désigne officiellement le fait qu'ils s'expriment dans une langue qui n'est pas le français scolaire. La circulaire d'octobre 2012 n'écarte pas la référence au parcours migratoire récent dans la régulation de l'affectation au dispositif UPE2A (Armagnague-Roucher et al., 2018), censé familiariser les élèves primo-arrivants avec le français en tant que "langue de scolarisation ", tout en introduisant de la souplesse dans la ségrégation scolaire imposée jusque-là aux élèves primo-arrivants.

Cet article interroge les finalités ainsi que les modalités d'action d'un dispositif scolaire (Barrère, 2013) à partir de ses dénivèlements, des formes latentes et plus modérées au sein de l'allophonie. II permet également de mener une réflexion sur la reconfiguration des frontières au sein de l'espace européen à partir des politiques éducatives linguistiques qui se jouent au sein du système éducatif français. Cela nécessite d'interpréter en tant qu'effet spécifique de frontière le fonctionnement d'une catégorisation qui gouverne, filtre et hiérarchise une population scolaire biopolitiquement définie par une circulaire relevant de l'infra-droit (Lochak, 1985 : 205). Comment une expérience de recherche ethnographique sur la scolarisation des mineurs étrangers peut-elle contribuer au champ des théories afférentes aux border studies?

La frontière dont il est question dans cet article oscille entre une dimension symbolique et sa matérialisation. La catégorisation de l'allophonie produit des effets de confins, au sens d'une séparation. Une telle catégorisation produit également une multiplication de différences et une constellation de statuts à l'intérieur d'une population scolaire présumée plus ou moins homogène (les «allophones") ainsi qu'une séparation entre ce groupe et les élèves francophones de classe ordinaire. L'espace matériel du dispositif UPE2A au sein duquel ces processus de segmentation et de ségrégation spatio-temporelle sont produits ne peut être considéré comme une scène neutre, autrement dit le théâtre d'une action sociale. En effet, il s'agit d'un espace " de moins en moins neutre, de plus en plus actif, à la fois comme instrument et comme objectif, comme moyen et comme but. Ce qui outrepasse singulièrement la catégorie dans laquelle on l'emprisonne, le "medium" " (Lefebvre, 2000 : 472). Pour cette raison, nous prendrons appui sur une notion empruntée à la géographie humaine et que Ratzel (2017) nomme Grenzsaum, au sens d'une bande de frontière ou bande de contention que nous mobilisons pour définir l'espace spécifique de I'UPE2A où ces processus de segmentation sociale sont re-produits. L'idée de Grenzsaum conduit à dépasser toute métaphore linéaire et binaire de la frontière (Balibar, 2015 : 93 sq.), permettant d'appréhender la complémentarité entre la dimension

5 Cf. les deux circulaires antérieures : n²002-063 du 20/03/2002 et n²002-100 du 25/04/2002. 
symbolique de la frontière et sa contrepartie spatiale au sein du dispositif étudié. En ce sens, cet espace ségrégatif spécifique peut être interprété comme une " abstraction concrète $"{ }^{6}, c^{\prime}$ est-à-dire " le devenir-objet d'un concept " (Virno, $2001: 40)$.

Dans la première partie de notre article, nous situons l'allophonie dans le cadre de politiques d'immigration fondées sur la "langue du tri " (Lochak, 2013), en dégageant sa dimension biopolitique. En l'occurrence, cette dimension renvoie dans notre démarche à la catégorie institutionnelle d'allophonie en tant que dispositif de frontière spécifique. Celui-ci produit une fragmentation spatiotemporelle contribuant à définir de nouveaux espaces, de nouvelles hiérarchies et de nouvelles relations de pouvoir au sein de l'institution scolaire française. Dans la seconde partie de cet article sont présentées les modalités par lesquelles ces élèves accèdent à l'école française, puis les expériences qu'ils en font au quotidien.

\title{
Encadré 1 : Méthodologie
}

\begin{abstract}
Cette recherche prend appui sur une démarche qualitative et ethnographique. Elle s'est centrée sur les dispositifs UPE2A des établissements scolaires de quatre différents territoires de l'Eurométropole. Dans chacun de ces établissements, Simona Tersigni a travaillé d'abord seule, puis avec Lorenzo Navone pour réaliser des entretiens et des observations au cours de deux années scolaires. Trente-sept entretiens ont été menés auprès des enseignants des dispositifs, des équipes de direction, de la vie scolaire, des psychologues de l'éducation nationale, des personnels des centres d'information et de documentation (CDI), des infirmières et assistantes sociales, de quelques cadres de l'Éducation nationale à l'échelle académique et départementale ainsi que du Centre d'Information et d'orientation (CIO). Ces données ont été complétées par des observations et des entretiens conduits auprès d'acteurs associatifs (médiation culturelle, centres socio-culturels, CADA) ${ }^{7}$ et $\mathrm{d}^{\prime}$ acteurs syndicaux liés à l'enseignement, mais également avec huit élèves ${ }^{8}$ insérés en UPE2A ou l'ayant fréquentée durant l'année qui a précédé l'entretien. Cet article prend appui notamment sur l'ethnographie qui s'est déroulée dans deux collèges. Le premier se trouve à proximité du centre-ville, dans un quartier accueillant notamment la classe moyenne locale. Le second collège est situé
\end{abstract}

6 II s'agit d'un concept introduit par Karl Marx dans les Manuscrits de 1844, afin de définir la dimension abstraite et matérielle de l'argent et du travail. Les phénomènes qui dans les Manuscrits étaient subsumés sous la catégorie d'aliénation (misère ouvrière, formes de dépossession) se retrouvent filtrés, requalifiés et subordonnés au contenu d'une analyse de l'exploitation du travail dans la théorie de I'argent (Haber, $2008: 31$ ) dans Le Capital (livre I, chapitre III) : " I'aspect de la monnaie ne trahissant point ce qui a été transformé en elle, tout, marchandises ou non, se transforme en monnaie. [...] Rien ne résiste à cette alchimie, pas même les os des saints, et encore moins des choses sacro-saintes, plus délicates ".

7 Dans les Centres d'accueil pour les Demandeurs d'Asile de Strasbourg, Mulhouse, Ferrette et alentours, Simona Tersigni a également travaillé avec Anaïk Pian tout au long de l'enquête IMSODA (2016-2017) : le croisement entre ces deux terrains a produit des matériaux intéressants qui seront évoqués seulement en partie dans le premier paragraphe de cet article (cf. Pian et al., 2018).

8 Tous les entretiens avec les élèves en provenance d'Italie (en nombre de six) ont été menés en italien par les auteurs de cet article. 


\begin{abstract}
dans la périphérie nord de la ville et est classé réseau d'éducation prioritaire. L'ethnographie scolaire durant les heures du dispositif UPE2A ainsi qu'en classe ordinaire et les entretiens retraçant la trajectoire migratoire, résidentielle et de sociabilité extra-scolaire, ont permis de mieux voir comment des catégorisations scolaires apparaissent, s'atténuent et resurgissent. Les observations ont été réalisées dans des situations et lieux centraux de la vie scolaire ordinaire : en classe (au moins une demi-journée par semaine dans chaque établissement), dans la cour de récréation, à la cantine, lors des sorties scolaires, lors des spectacles organisés à l'intérieur des établissements et lors de la semaine " école ouverte » au printemps 2016. Les auteur.e-s de cet article - qui sont également parents délégués ou en contact avec les associations d'Italiens strasbourgeois, réalité socialement segmentée - ont par ailleurs chacun'e des enfants scolarisés dans les sections internationales. II s'agit d'une filière publique que les élèves interviewés ne connaissent pas nécessairement. Certain'e-s d'entre eux ou leurs frères ou sœurs sont susceptibles d'y accéder dans les années à venir, sans que cela ne se fasse automatiquement. De la maternelle à la terminale du lycée strasbourgeois concerné par la section internationale d'italien, sont scolarisés des élèves italiens, des descendants d'immigrés italiens et, de manière croissante, des élèves " maghrébins " nés ou antérieurement scolarisés en Italie ${ }^{9}$. Leurs trajectoires racontées dans des textes écrits en préparation du baccalauréat confirment les récits des "UPE2A italiens " que nous avons rencontrés.
\end{abstract}

\title{
La biopolitique de I'allophonie
}

Entre les années 1950 et 1960, les deux sociologues William Herberg (1960 [1955]) puis Milton Gordon (1964) pointent la place secondaire de la religion dans la conception de l'assimilation états-unienne, en mettant davantage l'accent sur d'autres critères dont la maîtrise de la langue nationale. Si la conversion religieuse n'est pas requise aux migrants qui arrivent alors aux États-Unis, en revanche, ces derniers font l'objet d'une liste d'injonctions relatives notamment à l'apprentissage de l'anglo-américain ${ }^{10}$, à l'adoption de la culture ambiante, si ce n'est pas l'acquisition de la nationalité américaine. Dans la même période, I'anthropologue norvégien Fredrik Barth (1995 [1969]) défend la prise en compte de l'expérience du quotidien dans la constitution des processus génératifs des frontières ethniques, dont les marqueurs linguistiques font également partie. L'analyse sociologique du lien entre l'assimilation et la langue nationale aux États-Unis tout comme la mobilisation du critère linguistique dans le cadre des relations interethniques, n'ont pas pour autant nourri une pléthore de travaux en sciences sociales permettant de situer l'allophonie dans le contexte européen contemporain. Ils n'ont pas non plus suscité des publications visant à saisir

9 À l'approche des dix-huit ans, leurs sœurs ou frères aînés sont rarement scolarisés : ils doivent se contenter souvent de cours de français dispensés dans les centres-sociaux de Strasbourg ou de pratiquer le go-between avec la ville italienne où elles/ils résidaient avant de s'installer en France.

1012 millions de migrants sont passés par l'île d'Ellis Island entre 1892 et 1954, ce qui impliquait une période de quarantaine et de visites médicales, suite à laquelle les adultes comme les enfants devaient passer plusieurs tests mesurant la maîtrise de la langue anglo-américaine à partir d'une batterie de questions. Cf. https://ntieva.unt.edu//pages/ about/newsletters/vol_11/issue1/questions.htm 
des formes de singularités relatives à des choix biopolitiques (Foucault, 2001 et 2004 ; Macmillan, 2010). Pourtant ces politiques linguistiques renvoient à des technologies de pouvoir, correspondant à la rationalité gouvernementale libérale ainsi qu'à la gestion souple des phénomènes de masse associés à la population. II s'agit bel et bien d'une déclinaison du biopouvoir ${ }^{11}$ centrée sur des "prestations productives "(Foucault, 1994). Celles-ci impliquent " une véritable incorporation du pouvoir, en ce sens qu'il a dû arriver jusqu'au corps des individus, à leurs gestes, à leurs attitudes, à leurs comportements de tous les jours; de là l'importance de procédés comme les disciplines scolaires qui ont réussi à faire du corps des enfants un objet de manipulations et de conditionnements très complexes $"{ }^{12}$. La discipline de l'apprentissage linguistique est pensée comme une prémisse indispensable à l'intégration dans le cadre d'un déterminisme social considéré comme inéluctable. Concevoir la ségrégation du dispositif scolaire créé ad hoc pour les enfants migrants comme le moyen de l'intégration revient à créer des sujets subalternes : la norme par laquelle le biopouvoir s'impose, exerce une pression insistante et continue qui favorise la docilité et l'utilité des corps du " bon élève migrant".

Notre recherche se situe dans le contexte économique, historique et politique européen actuel, marqué par une crise générale (Giglioli et al., 2017) : une crise politique (Brexit; menace relative aux deux piliers de l'UE - l'espace de libre mouvement dans I'aire de Schengen et la monnaie unique dans l'euro-zone ; une émergence partout en Europe des partis d'extrême droite, populistes et xénophobes); une crise économique et financière dans les pays les plus vulnérables de l'Union et une ultra médiatisation de la " crise " migratoire en crescendo (à partir de la période comprise entre le Printemps arabe et 2015). Depuis plusieurs années, les sciences sociales analysent la "dimension productive " de la crise, en tant que moment violent de "rupture " d'un ordre précédent plus stable, tout particulièrement pour ce qui est de la période qui a suivi les attentats du 11 septembre 2001 (Bigo et al., 2008). Reconnaître le rôle de l'Europe politique dans la "dimension productive " de la crise (New Keywords Collective, 2016 : 10 et 21), revient à souligner qu'il y a une redéfinition de la relation entre liberté, démocratie et sécurité. Cette redéfinition s'opère souvent au détriment des deux premiers éléments par le recours à une rhétorique de la crise elle-même qui justifie et accélère la mise en place de politiques sécuritaires.

Dans la situation européenne contemporaine, les discours et les sonnettes d'alarme portant sur le croisement entre ces différentes " crises " (économique, politique et " humanitaire ") dictent sans cesse les règles pour l'admission

11 La biopolitique renvoie à la transformation d'un certain nombre d'éléments de nos vies en enjeux du pouvoir : "Par biopolitique, on semble malgré tout se référer dans tous les cas à un type particulier d'exercice du pouvoir (ou à un type d'intervention des dispositifs politiques) portant sur des objets spécifiques, et que l'on pourrait caractériser au sens littéral comme des "biopouvoirs", c'est-à-dire comme une application des pouvoirs à la vie " (Revel, 2013).

12 Issue d'un entretien paru d'abord en italien en 1977 (Microfisica del potere,Turin, Einaudi, p. 19), cette citation a été insérée plus tardivement dans une publication en langue française (Foucault, 1994) et est également accessible en ligne: http://1libertaire. free.fr/MFoucault134.html 
dans l'espace européen ${ }^{13}$. Elles fixent également la suspension intermittente de la liberté de mouvement dans l'espace Schengen. La crise, dans ses multiples formes, peut désormais être interprétée comme l'élément crucial de la contemporanéité politique (Agamben, 2013). Dans ce cas, est-il possible d'en observer un effet subreptice dans la création de nouveaux critères d'identification et de catégorisation, à l'instar de l'allophonie et des espaces comme I'UPE2A, impliquant un confinement spatio-temporel à l'intérieur de l'institution scolaire française?

\section{Les politiques linguistiques en Europe : des adultes aux enfants migrants}

La modernité politique n'est plus caractérisée par un équilibre entre des techniques de coercition et une idéologie du laisser-faire, mais plutôt comme un ensemble de techniques susceptibles de moduler, de différentes manières, une même exigence de coercition des corps dociles (Foucault, 1997, 2001 et 2004). Par conséquent il importe de regarder de près les politiques linguistiques en situation migratoire ainsi que les modalités d'enseignement du français destiné à des étrangers. Leur lecture nous fournira d'autres outils pour appréhender le fonctionnement des catégorisations associées aux langues d'autrui voire à leur " sonorité ", comme c'est le cas dans le terme d'allophonie, dès lors qu'on se déplace du côté des enfants et jeunes migrants.

À l'échelle européenne, I'Union européenne se focalise sur des politiques linguistiques pour l'enseignement des langues européennes ${ }^{14}$ alors que le Conseil de l'Europe (COE) promeut une articulation entre les cultures et les langues, à travers des recommandations finalisées énonçant les principes qui régissent les interventions politiques dans le domaine migratoire (COE 2014). L'intégration linguistique des migrants adultes a fait l'objet de trois rapports du Conseil, dont le dernier (COE 2014) pointe que dans la période comprise entre 2007 et 2013, la maîtrise de la langue du pays d'immigration (à partir de test de langue, entretiens avec un agent de l'immigration ou présentation de certificats et diplômes par les migrants) est requise dans la plupart des pays du Conseil pour l'entrée sur le territoire national ainsi que pour l'obtention d'un titre de séjour ou de la naturalisation ${ }^{15}$. Des conventions ainsi que des recommandations du Comité des ministres et des recommandations de l'Assemblée parlementaire incluant les travailleurs migrants et les membres de leurs familles ainsi que leur éducation et développement culturel composent le référentiel normatif en vigueur.

À l'échelle de la France, les critères gouvernementaux d'intégration pour les migrants datent de la seconde moitié des années 1990 (Marie, 1996). C'est également le moment où les formations universitaires en français langue

13 II s'agit de nouveaux accords bilatéraux avec des États tiers pour externaliser la gestion des frontières de I'Union européenne, de la réorganisation de l'agence Frontex, désormais en charge des frontières européennes et des côtes, de la création des hotspots notamment.

14 Les chiffres clés de l'enseignement des langues à l'école en Europe (Eurydice, 2005, 2008 et 2012).

15 Des nuances sont cependant repérées dans ce rapport entre les pays du Nord, de l'Est et du Sud de l'Europe politique. 
étrangère (FLE) sont instituées. Cependant, l'introduction des critères linguistiques comme élément de justification de la sélection opérée à l'égard des migrants est plus tardive. La langue française est centrale dans les formations linguistiques et civiques organisées dans le cadre du contrat d'accueil et d'intégration (CAI), ce qui a fait l'objet de recherches importantes (Hachimi Alaoui, 2014 ; Gourdeau, 2016). Puis, plus récemment, en lien avec le contrat d'intégration républicaine $(C I R)$ ainsi que dans le cadre de la demande d'asile (français langue d'accueil), la langue française a acquis une place considérable dans les politiques éducatives pour les jeunes migrants ${ }^{16}$.

Entre 2004 et 2005, deux rapports parlementaires ${ }^{17}$, rédigés dans le cadre des travaux de la commission Prévention du Groupe d'étude parlementaire sur la sécurité intérieure, se consacrent à la fabrication d'un nouveau sujet déviant : le " migrant bilingue " (et ses descendants). Le rapport préliminaire établit un lien causal direct entre le bilinguisme précoce (ayant trait à la trajectoire migratoire familiale), l'échec scolaire et la délinquance. Tout en évitant les formulations présentes dans le pré-rapport, le rapport définitif " cherche ainsi à éviter une mise en relation trop directe " entre le bilinguisme et la délinquance (Muni Toké, 2009 : 36). Le débat sur le " bilinguisme pathogène " suscité par les deux rapports s'inscrit dans la construction de l'autochtonie (Détienne, 2003 : 106), entendue comme un processus qui " se fonde et refonde continument ". À l'instar de ces multiples définitions d'autochtonie, il est possible de nuancer l'allophonie, en montrant les dénivèlements dont sont captifs les élèves étiquetés comme allophones, dans un espace plus spécifiquement scolaire. II existe en effet différents degrés d'allophonie " en termes d'assignation et de ressenti allant de I'allophonie tempérée à I'allophonie totale " (Armagnague-Roucher et Tersigni, 2019) : la première implique " une forme latente plus modérée et moins explicite d'une même dynamique de segmentation sociale de l'univers scolaire " tandis que la seconde renvoie à une figure absolue de l'étrangeté en termes d'altérisation extrême (Ibid.). L'allophonie tempérée se constitue comme une "zone grise " où se regroupent différentes expériences scolaires impliquant pour les élèves allophones tantôt des attentes et des gratifications, tantôt des formes de disqualifications, allant de l'indifférence jusqu'à des sanctions directes et indirectes. Ainsi dans le cas de l'allophonie tempérée, qui est de loin la plus " problématique ", des trajectoires scolaires sont susceptibles d'être fortement surdéterminées. Faut-il alors s'étonner que l'allophonie n'ait pas suscité de fortes oppositions après octobre 2012, au moment de la circulaire qui I'a instituée ${ }^{18}$, ni après ?

16 À titre d'exemple, la région Grand Est a créé une plateforme de ressources pour la maîtrise du français et des compétences de base (PARcours et Orientation Linguistique, PAROL). Cf. www.parol-grandest.fr

17 Cf. Bénisti Jacques-Alain (Dir.) (2005) Rapport de la Commission Prévention du Groupe d'études sur la sécurité intérieure. Sur la prévention de la délinquance. Rapport définitif, Assemblée nationale, XIle Législature. II s'agit d'une version rectifiée par rapport à celle diffusée en 2004 (Muni Toké, 2009).

18 Voir par exemple : "Le recours à la catégorie "allophonie" peut apparaître comme une avancée pour un certain nombre de didacticiens et de sociolinguistes dont je fais partie »(Belondo, $2016:$ 452). 


\section{L'allophonie tempérée dans une capitale européenne}

Pour un certain nombre d'élèves migrants, dépositaires d'un faible capital scolaire, la possibilité de bénéficier d'une " classe cocon " au sein du dispositif UPE2A représente un avantage certain. Plusieurs chercheurs et professionnels du secteur ont dû estimer préférable de ne pas déconstruire la catégorie d'allophonie pour " ne pas tirer sur la croix rouge ", au sens de ne pas prendre le risque de déclencher un acharnement sur une "cible " déjà en souffrance, du côté des élèves comme des enseignants. Quatre ans après la diffusion de la circulaire instituant la figure des EANA, cette catégorie a fait l'objet d'appréciations globalement positives sur le plan de la didactique de la langue française (Belondo, 2016 : 451-452). En revanche, du point de vue de la didactique des langues, deux bémols sont introduits. D'une part, le manque de reconnaissance à l'égard des compétences plurilingues des jeunes dits allophones est considéré problématique (Ibid.). D'autre part, des doutes sont exprimés quant à l'emploi effectif de ce terme dans des situations de classe et d'établissement : la manière courante de nommer ces élèves allophones dans les établissements n'a pas directement recours à I'allophonie. "UPE2A ", à savoir le nom du dispositif dont bénéficient les élèves dits allophones, est le sigle employé, au pluriel, pour désigner les élèves relevant de ces dispositifs. Cette formulation émique (du point de vue des personnels scolaires) ${ }^{19}$ engendre celle d' "UPE2A italiens " figurant dans le titre de cet article : elle s'applique à nos jeunes interlocuteurs d'ascendance nord-africaine ayant migré à Strasbourg.

Peut-on encore parler d'un marqueur linguistique dès lors que la catégorie mobilisée par l'éducation nationale (allophone) contourne les mots qui, au cours de l'histoire occidentale, ont désigné un système d'expression propre à un groupe ${ }^{20}$ ? Si le terme d'allophone s'est imposé sur celui d'" alloglotte " pourtant existant ${ }^{21}$, il convient de ne pas évacuer ce qui se dissimule derrière l'étymologie de l'adjectif allophone, à savoir la " sonorité d'autrui ". Plus que de la reconnaissance d'autres langues ou idiomes, donc expressions avec un sens collectif, ne s'agirait-il pas d'une sorte de bruit de fond?

L'emploi de ce terme ne fait qu'illustrer la tendance des majoritaires à s'identifier implicitement à l'ordre de l'universel (Guillaumin, 1972) y compris sur le plan linguistique. C'est, d'ailleurs, de l'ordre de l'impensable que des spécialistes de I'enseignement du français langue seconde (FLS) se mettent à employer le terme d'" autophonie " pour désigner, en miroir de l'allophonie, la catégorie des élèves pratiquant le français scolaire de la classe ordinaire.

Les élèves "UPE2A italiens " se situent précisément entre la vaste catégorie officielle de l'allophonie et celle des élèves maîtrisant le français scolaire. Sur le plan de I'hétéro-désignation, ce sont en quelque sorte des " allophones de l'inté-

19 Le terme " émique " désigne le point de vue de l'acteur, alors que le terme "étique " désigne le point de vue extérieur, ici en l'occurrence, celui du chercheur et de la chercheuse (Olivier de Sardan, 2008).

20 II s'agit notamment de logos, glôssa et idiôma en grec ; eloquium, lingua, loquela, idioma, locutio, sermo, oratio en latin ; idiome, langage, langue, parole en français (Cassin et al., $2004: 675-676$ ).

21 II a pourtant été proposé dans le paysage scientifique (Armagnague-Roucher et Tersigni, 2019). 
rieur ", puisque le critère pris en compte est leur intériorité à l'Europe politique par le système scolaire qui les a formés antérieurement. Nous employons ad hoc cette expression qui recèle en elle quelques ambiguïtés, étant donné la proximité avec celle de l'u ennemi intérieur " (Rigouste, 2007). À l'instar de la méfiance qui existe à l'égard de cette figure en terme de menace pour l'ordre démocratique, politique et démographique, I'" allophone de l'intérieur " est susceptible de déclencher des inquiétudes quant à I'homogénéité des compétences des élèves en classe ordinaire et en dispositif. Dans le cas de I'" ennemi intérieur ", l'ordre public se trouve mis en cause, dans celui de l'" allophone de l'intérieur ", c'est l'ordre scolaire qui se trouve mis à mal.

Cette dernière expression permet de souligner combien ces élèves, qui ne sont pas considérés immédiatement comme des " inopportuns scolaires "22, ne sont pas non plus prioritaires dans l'individualisation de l'accompagnement à l'intérieur du dispositif, ce qui pourrait engendrer, au final, des mauvaises surprises en termes de réussite et de conduite scolaire. Labélisés en tant $q u^{\prime}$ " italiens " puisqu'italophones au moment du test d'entrée à l'école publique, notamment pour ce qui est des collégiens, ces élèves sont évalués par des psychologues au $\mathrm{ClO}$. Cette évaluation associe un critère linguistique officiel issu $\mathrm{d}^{\prime} u n$ test écrit ${ }^{23}$ au fait d'être des élèves primo-arrivants ayant bénéficié d'un système scolaire européen considéré favorablement en France. Toutefois, leur capital scolaire n'est pas garanti, ni nécessairement valorisé. Pour rejoindre une formation au sein de laquelle certains italophones sont présents depuis le début de leur scolarité, ces élèves "italo-maghrébins " devront faire preuve d'engagement scolaire, avec notamment de bons résultats en mathématiques, tout en apportant la preuve du maintien de la maîtrise de la langue italienne.

Pour comprendre cette catégorie d'allophonie tempérée, il importe de déconstruire les mécanismes dans lesquels elle est produite et ses effets en terme de segmentation entre trois types d'élèves : les allophones dont les langues de scolarisation sont considérées plus éloignées du français ; les élèves censés maîtriser le français scolaire ${ }^{24}$ et, enfin, les élèves maîtrisant d'autres langues européennes, en l'occurrence I'italien scolaire. À Strasbourg, une partie de ces derniers peut être légitimement polyglotte, grâce à l'existence des sections internationales. Les élèves "italo-maghrébins " restent souvent en établissement de secteur, sans parvenir à accéder à la filière internationale par manque de renseignements à leur arrivée ou parce que la passation du test des sections internationales peut s'effectuer sans qu'ils en aient compris l'enjeu, par manque de précisions de leurs enseignants. Par conséquent ils ne rentrent pas tout à fait dans ce troisième cas de figure.

22 Terme issu de la notion de "postérité inopportune " développée par Palidda (2011: 10) à partir des réflexions de Sayad (1999, notamment).

23 Ce test prouve la compréhension de I'italien par un petit QCM et par la rédaction de phrases dans la même langue.

24 Cette catégorie n'est pas homogène du point de vue social ni culturel, incluant tantôt des familles qui ne sont pas associées à un passé migratoire tantôt les descendants de migrants. 


\title{
Encadré 2 : L'enseignement de la langue italienne à Strasbourg
}

\begin{abstract}
L'enseignement de la langue et de la culture italienne à Strasbourg est assuré dans différents lieux et institutions. Pour les jeunes en âge scolaire, il faut mentionner les ELCO, dont la création en 1973 a précédé de quelques années celle des sections internationales de l'école publique (de la maternelle jusqu'au baccalauréat) qui date de 1980 ainsi que l'enseignement en tant que quatrième langue au sein de l'école européenne, à partir de la quatrième année, puis en tant que LV2 dans quelques collèges publics. D'autres initiatives associatives dépendent en outre de I'association Caffè Italia Off (théâtre en italien pour les élèves de la fin du primaire et du collège, cours ludiques d'italien dans le cadre des activités d'un centre socioculturel d'un quartier socialement mixte) ou du Patronato Acli25 (cours de langue). Pour les adultes, l'enseignement est pris en charge par I'Institut italien de culture, par I'Université publique ainsi que par des formations en entreprise relevant des droits individuels à la formation (DIF). II existe enfin des initiatives associatives relevant de I'Université européenne populaire et de nombreux cours particuliers. À la différence de Metz, à Strasbourg ne sont présents ni la Società Dante Alighieri (organisme national pour l'enseignement de la langue et culture italiennes en Italie et à I'étranger) ni les COASCIT (des émanations locales des consulats italiens). Ces derniers visent des apprenants à large spectre qui ne sont pas nécessairement les résidents italiens à l'étranger ni les Français d'ascendance italienne, voulant garder un lien avec la langue et la culture de leurs ancêtres. L'enseignement de la langue italienne en section internationale à Strasbourg est, dans tous les cas, le produit d'accords entre le Rectorat et le Consulat de Metz. La politique consulaire pour ce qui est de l'enseignement de l'italien à Strasbourg se différencie ainsi de manière substantielle par rapport à d'autres centres urbains avoisinants de l'autre côté du Rhin, à l'instar de Fribourg ou Stuttgart. Dans ces dernières villes, la présence d'un consulat italien et I'absence des sections internationales d'italien au sein de l'école publique permettent aux COASCIT de jouer un rôle central dans ce secteur, contrairement à la configuration strasbourgeoise.
\end{abstract}

S'ils font figure ${ }^{26} \mathrm{~d}^{\prime}$ " allophones de l'intérieur ", c'est aussi parce que ces élèves qui se disent souvent " italo-marocains " ne sont pas nés italiens juridiquement, à l'exception de ceux dont I'un des parents est italien (la loi italienne sur la nationalité étant constituée sur le principe du Jus sanguinis). Par ailleurs ils ne peuvent aisément le devenir actuellement, en raison de l'échec du projet de loi de naturalisation qui n'a pas abouti à la fin de l'année $2017^{27}$ et dans lequel un poids important était attribué aux années de scolarisation en Italie. Nombreux sont les adolescents qui, parmi ces "UPE2A italiens ", sont nés en Italie et n'ont pas connu d'autres mobilités internationales avant de s'installer

25 Depuis 1945 ce réseau social de professionnels liés aux Associations chrétiennes des travailleurs italiens œuvre en Italie et dans vingt-deux autres pays du monde en soutien aux citoyens catholiques, avec différentes activités qui vont de la consulence psychologique à la formation continue.

26 II s'agit d'un jeu de mots avec le titre de l'ouvrage de Cossée et al. (2004).

27 Cf. http://www.lemonde.fr/europe/article/2017/12/28/en-italie-la-bataille-perdue-dudroit-du-sol_5235224_3214.html (consulté le 22/11/2018). 
en France ${ }^{28}$. Le flottement de leur allophonie tempérée peut les conduire du côté d'un bilinguisme, voire d'un plurilinguisme, discrédité - tel qu'il a été conçu dans les deux versions successives du rapport Bénisti, au sujet des descendants de migrants - ou alors du côté de la reconnaissance d'une compétence.

\section{" Vous aurez compris que j'ai migré une seule fois "}

L'allophonie tempérée est une catégorisation implicite, agissant à l'intérieur de l'institution scolaire française et elle s'insère à double titre dans la reconfiguration contemporaine des frontières internes à I'Union européenne sur le plan symbolique. D'une part, elle relève de la hiérarchisation des statuts migratoires, d'autre part, elle renvoie à la plus ou moins grande facilité d'intégration que sont censés rencontrer des groupes plutôt que d'autres. Les marqueurs linguistiques n'ont certainement pas remplacé d'autres facteurs d'établissement des frontières. Cependant, nous avons constaté que les élèves rencontrés veillaient tout particulièrement à évacuer de leurs récits toute référence aux croyances et aux pratiques relatives à l'islam, sauf dans quelques rares exceptions. En revanche, en Italie, I'hétéro-définition de " jeunes musulmans " s'accompagne souvent, pour une grande partie de ces jeunes, d'affichages publics tantôt " ethniques ", tantôt " italiens " de leur islamité (Trucco, 2015).

L'existence de la catégorisation française de "UPE2A italiens " et de la catégorisation italienne de " jeunes musulmans " renvoie à une juxtaposition d'étiquettes au sein de l'Europe politique. Nous ne sommes pas systématiquement en présence d'un " effet-écho "(Balbo, 1998 : 100), susceptible d'harmoniser des hétéro-désignations, pesant sur les migrants à l'intérieur d'une "Europe forteresse ". Cette image d'étanchéité produite par le recours au terme de forteresse occulte en effet le travail de filtrage, sélection et segmentation opérés continuellement par les frontières internes et externes de I'UE à l'égard des candidats à l'immigration et à l'asile. Dans ce contexte, l'approche barthienne de la frontière ne permet pas, dans sa dimension linéaire, de saisir pleinement les jeux d'" inclusion différentielle " (Mezzadra et Neilson, $2012: 67$ ) que nous avons observés empiriquement avec ces élèves "italo-marocains " dans l'espace ségrégatif du dispositif UPE2A.

Dans notre recherche, la trajectoire des élèves concernés par des " mouvements secondaires " de I'Italie à la France et les rapports de pouvoir dans lesquels ils sont pris, ont émergé à l'intérieur d'une frontière dont la configuration en tant que ligne se révèle insatisfaisante. Analyser l'émergence de marqueurs linguistiques propres aux élèves qui font figure d' " allophones de l'intérieur ", nous a conduits à analyser tout d'abord un rite d'institution (Bourdieu, 1982) qui les produit définitivement en tant qu'allophones d'un certain type (II.1), puis à relire I'espace scolaire en tant que frontière (II.2), au prisme du concept de " bande de

28 Voir par exemple le texte écrit par une élève " italo-marocaine " dans le cadre de la préparation au baccalauréat de section internationale d'italien : "Vous aurez compris que j'ai migré une seule fois [de I'Italie à la France], même si, à vrai dire, dans le passé ma famille décida de se réinstaller au Maroc, ce qui dura seulement six mois. Ce fût précisément dans ce dernier pays que naquirent mes parents, tous les deux originaires de la ville de Casablanca ". 
contention " (Ratzel, 2017) ${ }^{29}$.

\section{Produire les « UPE2A italiens "}

La notion de frontière à laquelle nous nous référons ne se configure pas comme une ligne permettant de séparer nettement un espace du dedans et un espace du dehors. II s'agit d'un ensemble de pratiques, de rites et processus d'inclusion et exclusion qui se reproduisent en cascade, tout en fragmentant l'espace et le temps. En ce sens, la frontière n'est pas un espace-temps de substitution. Elle produit ses propres sas spatio-temporels : ces derniers sont par ailleurs susceptibles de se multiplier par fragmentations successives. La catégorisation institutionnelle d' "UPE2A italiens" est le résultat d'une fragmentation à l'intérieur d'une étiquette plus large garantissant l'exercice du droit d'accès à un dispositif conçu pour l'ensemble des élèves catégorisés en tant qu'allophones. Certains de ces adolescents ne voient pas nécessairement d'un mauvais œil la catégorisation scolaire qui peut même paraître initialement rassurante et accueillante. Elle déclenche en effet un droit à la scolarisation, bien que ségréguée, facilitant pour ces adolescents une prise de recul face au dépaysement qu'ils vivent initialement. Sur le plan subjectif et personnel, ces adolescents se retrouvent devoir faire le deuil (du pays quitté) qu'impose une migration internationale, alors que seuls leurs parents (et pas la famille toute entière) font l'expérience d'un " mouvement secondaire ". Qui plus est, dans la plupart de leurs trajectoires nous avons rencontré une dynamique familiale partagée. Ce n'est pas parce que cela fait désormais une dizaine d'années, depuis le début des années 2010, qu'en alternative au retour au pays d'origine, de nombreuses familles nord-africaines ont fait le choix de migrer dans des villes françaises, que ces jeunes sont mentalement préparés au changement qui les attend. Ce nouveau projet migratoire parental (et l'autonomie dont il faut faire preuve à l'égard des situations politiques et économiques en Europe) ne s'élabore pas avec les enfants. Ces derniers ne participent pas au choix d'une mobilité intra-européenne : celle-ci n'a pas fait l'objet de négociations familiales, ni même de véritables conversations familiales leur permettant de se préparer à leur nouvelle vie. Certains de ces adolescents, comme les frères Tareq et Mohamed, se retrouvent à Strasbourg, alors qu'ils croyaient déménager au Luxembourg ou bien à Paris.

" - [Tareq (quatorze ans, arrivé du Piémont — ville d'Asti — début juillet 2016)] : Je suis

arrivé d'Asti, qui se trouve près de Turin, je suis italien, je suis né là-bas, je suis d'origine marocaine. [...] Au début, je ne voulais pas venir, car je ne pensais pas que c'était une ville importante, je voulais aller à Paris. Ensuite, nous sommes arrivés ici en voiture avec toute la famille, un voyage de huit ou neuf heures, nous sommes arrivés et nous sommes allés directement à la maison. - [Chercheur] Pourquoi avez-vous " atterri " à Strasbourg? - [Tareq] Demande à mon père!

- [Mohamed] À vrai dire, je suis arrivé sans savoir que j'étais à Strasbourg. Je ne savais pas. - [Chercheur] Personne ne t'a expliqué que vous alliez vous rendre dans cette ville? - [Mohamed] À vrai dire je suis arrivé ici en voiture, je pensais arriver à Paris, mais en descendant de l'automobile, j'ai constaté que..."

29 Le recours à cet auteur peut paraître surprenant dans la mesure où, un de ses concepts, celui de Lebensraum (espace vital) a été instrumentalisé par les politiques expansionnistes nazi-fascistes après sa mort, qui remonte cependant à 1904. 
À l'instar de l'expérience migratoire des enfants de migrants étudiés par la sociologie italienne, pour ces adolescents arrivés à Strasbourg d'Italie le " regroupement des membres d'une même famille ne constitue pas l'inévitable étape finale du processus migratoire, d'autant plus qu'elle n'est pas toujours accompagnée par la cohabitation intergénérationnelle. Les regroupements des parents, des parents et de leurs enfants ou encore de tous les frères et sœurs peuvent être dissociés ou différés dans le temps, et des phases de séparation plus ou moins longues peuvent alterner avec des phases de cohabitation " (Miranda, 2012 : 67). Quoi qu'il en soit, le récit que Tareq et Mohamed font de leur l'arrivée à Strasbourg évoque un dépaysement certain. Dans un autre entretien, Fatima évoque l'attente avant d'être scolarisée en France, puis la prise de rendez-vous au $\mathrm{ClO}$ comme une issue en vue de son accès au collège.

" - [Chercheur] Et quand tu es arrivée en septembre, entre ton arrivée et ton premier jour d'école, combien de temps s'est-il écoulé ? Qu'est-ce que tu as fait entre-temps? - [Fatima] Pas mal de temps, je crois qu'il a fallu attendre même deux mois. Alors, je suis arrivée... [à Strasbourg], et nous avons appelé un centre administratif, un centre, je ne me souviens plus très bien..."

Le récit de Fatima sur son expérience au $\mathrm{ClO}$ de Strasbourg, donne cependant un aperçu clair d'un entretien qui doit évaluer les compétences scolaires alors que cette adolescente est encore mentalement en transit d'un pays à l'autre, ce qui ne lui facilite pas la tâche d'être à la recherche de son nouveau statut d'élève, en France.

" - [Chercheure] Te souviens-tu comment était le bâtiment et où il était situé ? Y avait-il une cour intérieure?

- [Fatima] C'est un centre qui n'est pas seulement un centre d'orientation, enfin, c'est aussi un centre d'orientation..., ils m'ont posé beaucoup de questions, ils m'ont aussi soumise à un test, j'ai évidemment répondu en français.

- [Chercheur] Et en quoi consistait-il ce test?

- [Fatima] : Ils m'ont demandé si j'avais étudié le français en Italie, si je parlais français, ils m'ont demandé d'écrire aussi un petit texte sur un sujet au choix, en français, l'important était d'écrire quelque chose pour qu'ils puissent voir si je savais écrire. Puis, ils m'ont demandé mon âge, l'âge de mon père et des informations de ce type.

- [Chercheur] Et puis ils t'ont demandé de montrer des papiers, par exemple les bulletins scolaires italiens?

- [Fatima] Oui, les bulletins scolaires. [...]

- [Chercheure] Et combien de temps cela a duré ? Une demi-heure?

- [Fatima] Peut-être un peu moins, sans doute vingt minutes, cela n'a pas pris beaucoup de temps."

Cet entretien relate un rite d'institution (Bourdieu, 1982) suite auquel Fatima, comme d'autres élèves, devient en quelque sorte une "UPE2A italienne " susceptible d'intégrer un collège, dès lors qu'une place en classe ordinaire se libère afin de garantir l'alternance avec le dispositif. Ici Fatima montre son usage de l'italien et du français, rend compte de son âge et donne la preuve de sa scolarisation antérieure par les bulletins italiens. En l'occurrence, ce rite d'institution fabrique implicitement du sens, justifiant que des élèves avec différentes trajectoires scolaires et linguistiques ainsi qu'avec différents âges se côtoient aussi longtemps dans un dispositif ségrégatif censé les faire progresser 
dans le français scolaire. Bourdieu (1986) avait bien différencié sa notion de rite d'institution de celle de rites de passage chez Van Gennep (1909) permettant notamment d'appréhender l'accès à un groupe d'âge supérieur. Or, dans cette situation se vérifie même une rétrocession en âge. Les plus âgés des élèves " italo-maghrébins " rencontrés ont souvent relevé la gêne éprouvée en raison d'une cohabitation d'âges au sein du dispositif (douze-seize ans). Certains de nos interlocuteurs vivent comme une injustice le prolongement de leur permanence en dispositif, au détriment de l'intégration dans la classe ordinaire, à laquelle ils aspirent en tant que " bons élèves ", comme il émerge de cet entretien collectif avec Mohamed et Tareq :

" - [Mohamed] La première fois, en classe ordinaire, j'ai fait seulement des maths. $\grave{A}$ vrai dire j'en fais encore... mais maintenant j'ai aussi du sport et de l'anglais en classe ordinaire, puis stop. J'ai reçu le dernier emploi du temps avant Noël, maintenant j'ai encore le même.

- [Chercheur] Et tu sais jusqu'à quand tu l'auras? - [Mohamed] Non, pas encore.

- [Tareq] La différence je l'ai ressentie parce qu'au début, durant le premier mois, j'avais que du français. Puis elle a commencé à ajouter les maths et l'anglais. Le dernier emploi du temps, elle me l'a donné il y a deux mois, il y a deux mois et demi, depuis il n'a pas changé. Pour rejoindre complètement la classe ordinaire, il me faut encore les sciences, puis histoire-géographie. Et aussi français avec la classe [ordinaire]. Je ne comprends pas pourquoi elle ne me place pas en classe ordinaire, j'ai tout bon. Elle a même fait passer en classe ordinaire un gars qui ne sait rien. "

La posture de Tareq, à l'instar de celle d'autres "UPE2A italiens " comme lui, laisse penser que le référentiel mobilisé dans le rite d'institution du $\mathrm{ClO}$ ne légitime pas pleinement ce qui se met en place, réduisant ce moment de rencontre institutionnelle à un acte bureaucratique, pourtant effectué par des psychologues.

\section{L'allophonie tempérée comme « bande de contention " scolaire}

Parmi ces élèves "italo-maghrébins ", plusieurs soulignent un flottement entre la nostalgie de la vie scolaire en Italie et l'appréciation du collège français, certes cadenassé dans ses règles à leurs yeux, mais permettant davantage de structuration. Ce balancement entre les deux constats ne permet pas de donner du sens ni à la nouvelle vie strasbourgeoise ni à une scolarité qui finit par prendre appui sur de nouveaux liens d'amitié. Les récits des trajectoires de ces élèves " italo-maghrébins " évoquent le contexte de départ d'Italie et d'arrivée en France, puis se centrent sur leurs affinités électives, celles entre des élèves à la fois " allophones " et d'ascendance nord-africaine, mais sans liens avec les descendants de migrants nord-africains de France. Fatima (quinze ans) est une jeune tunisienne née à Palerme. Installée à Strasbourg avec sa famille (puisque d'autres membres de la famille s'y étaient déjà établis), à l'instar d'autres collégiens arrivés d'Italie, Fatima met en évidence qu'à I'origine de cette décision il y a eu la crise économique italienne : en l'occurrence, son père avait perdu son emploi sans parvenir à en trouver un autre. Fatima a noué une relation d'amitié avec Sabrina, jeune Algérienne rencontrée en UPE2A, et avec Omar, arrivé de la banlieue napolitaine en même temps qu'elle. 
" - [Chercheur] et entre vous, est-ce qu'il vous arrive d'échanger, ne serait-ce que pour les devoirs?

- [Fatima] Alors, avec Omar oui. Nous nous sommes aidés mutuellement, mais pas avec les autres. Cela dépend du lieu de résidence, parce qu'Omar vient du Sud, comme moi, là-bas en Italie, il habitait à Naples et moi à Palerme et sincèrement les gens du Nord se la ramènent, donc je ne les trouve pas sympathiques... donc quand j'ai besoin de son aide, je lui envoie un texto alors qu'avec les autres je n'ai pas trop envie de parler ou alors seulement en raison des pays où "nous habitons". "

Les propos de Fatima montrent que ce n'est pas nécessairement la maîtrise de l'italien, pourtant relevée par le $\mathrm{ClO}$, qui structure le cadre des relations amicales entre ces adolescents marqués par une appartenance à plusieurs pays en même temps (les pays où " nous habitons ", au sens de la France, mais aussi de I'Italie, du Maroc, de la Tunisie et de I'Algérie). Fatima est d'ailleurs proche de Sabrina puisqu'elles " se racontent leur vie en arabe ", tandis qu'avec Omar, une proximité culturelle et affective s'impose en raison d'une intériorisation des " relations interethniques " italiennes ${ }^{30}$, entre le Nord et le Sud. Fatima comme Omar s'identifient au Mezzogiorno et dans ce cas, ils n'incluent pas dans leur sphère intime les "UPE2A italiens " arrivés des villes du Nord. Leur auto-définition ne colle pas avec l'hétéro-assignation institutionnelle implicite des allophones de l'intérieur, les "UPE2A italiens".

Les transitions scolaires de I'Italie à la France - que nous avons observées se frottent à des politiques éducatives fabriquant des sas spatio-temporels à l'endroit des enfants migrants. Ces politiques mettent l'accent sur les devoirs des élèves migrants et font fi du durcissement des politiques d'immigration et d'asile en Europe, ainsi que de la structuration des systèmes scolaires des pays d'origine des élèves allophones, y compris quand ils sont originaires de pays européens. L'arrivée dans le dispositif français UPE2A peut se révéler malaisée, même pour les jeunes "italo-maghrébins ». En plus des difficultés d'ordre linguistique, l'entrée dans le système éducatif français les oblige à se familiariser avec toute une série de normes et codes à leurs yeux incompréhensibles $^{31}$. Dans la mesure où le début de leur scolarité en France est caractérisé par une présence en dispositif qui limite fortement I'accès à la classe ordinaire, ces élèves, comme tous les EANA, partagent leur quotidien scolaire avec des élèves qui ne sont pas tout à fait des pairs, le décalage en âge pouvant même atteindre quatre ans. Ces éléments contribuent à faire surgir une nouvelle forme de dépaysement, comme le relate notre jeune interlocuteur ${ }^{32}$ :

30 La " question méridionale " correspond à la désignation italienne pour définir les relations interethniques entre le Nord et le Sud du pays depuis son unification en 1861.

31 Par exemple le cycle scolaire du collège qui est composé par un ordre à rebours dans I'appellation des différentes classes (sixième, cinquième, quatrième, etc.), le déplacement des élèves d'une classe à l'autre (au lieu de celle des enseignants), mais également la présence de toute une série de professionnels scolaires qui n'existent pas en Italie (surveillants, CPE, psychologues, infirmiers et assistants sociaux scolaires) dont le rôle n'est pas aisément déchiffrable initialement, mais qui peut être ensuite appréciable.

32 Tous les entretiens avec les élèves " italo-maghrébins " se sont déroulés en italien et en présence des deux auteur·e.s de cet article. Les verbatim sont tous traduits de l'italien en français. 
" - [Chercheur] Quelles impressions as-tu eues quand tu es arrivé en dispositif? - [Mohammed (quinze ans, arrivé de Vénétie en août 2016)] Et bah, quand je suis entré j'ai pensé : mais s'il y a autant d'enfants si petits, pourquoi suis-je avec eux? Mais finalement

j'ai compris pourquoi... Heureusement j'ai compris que je n'étais pas avec eux, au sens où j'y étais qu'en partie."

Pour Mohammed comme pour ses camarades de dispositif, l'étiquette d'allophone implique que de multiples disqualifications soient en jeu : le mélange d'âges y compris avec les sœurs et frères cadets, la rétrocession de degré scolaire (du lycée en Italie au collège en France) ainsi que le maintien dans l'espace de relégation qu'est le dispositif UPE2A. L'étiquette d'allophone implique souvent des processus de rétrocession sur le plan du degré scolaire (du lycée en Italie au collège en France), à l'instar de ce qu'en dit Fatima :

" - [Chercheur] Et tu te retrouves dans quelle classe? - [Fatima] En troisième, ce serait la dernière année du collège, alors que j'avais déjà commencé le lycée. - [Chercheur] Donc tu as perdu un an? - [Fatima] J'avais déjà passé mes examens de la fin du collège en Italie et cette année je dois le passer à nouveau durant l'été. - [Chercheur] Mais en même temps, pour la langue française...

- [Fatima] Puis on verra.

- [Chercheur] Mais tu avais déjà fait un an au lycée?

- [Fatima] Oui [...] et j'avais même été admise à l'année suivante, en revanche cette année je suis en troisième, et donc je ne sais plus si j'ai perdu un ou deux ans. - [Chercheur] Et par rapport à l'âge, comment tu te sens avec les camarades du collège qui n'ont pas vécu cette mobilité à l'étranger? - [Fatima] Hein, ce n'est pas grave l'âge, car ils ont presque tous quatorze ans, il est rare d'en trouver un qui a quinze ans, clairement seulement ceux qui ont doublé la quatrième, mais ce n'est pas une question d'âge. Nous sommes tous amis, surtout entre "Arabes", il est clair qu'entre nous on s'entend mieux."

La condition sociale et familiale de ces adolescents peut paraître plus stable et même privilégiée. Elle subit tout autant la production de processus de hiérarchisation au sein desquels le degré d'allophonie ainsi que le capital culturel sont une composante primordiale, au sein du dispositif. Un des effets d'une telle hiérarchisation se manifeste dans la distribution asymétrique, à l'intérieur du dispositif, d'une série de gratifications, d'attentes et de ressources. L'ensemble des étudiants inscrits dans un dispositif relève des mêmes droits, dans le cadre de ce qui pourrait juste paraître une sorte de discrimination positive ne serait-ce qu'en raison du nombre réduit d'élèves (ils ne dépassent pas le nombre de vingtquatre). Cependant, la perception qu'en ont les élèves qui en bénéficient ainsi que les attentes reliées à leurs droits diffèrent de manière substantielle, autant du point de vue de l'enseignante que des collégiens. Pour ce qui est des élèves " italo-maghrébins " en tout cas, l'alternance entre les heures de présence en dispositif et celles en classe ordinaire dessine une zone grise d'attente au sein de laquelle I'UPE2A, de lieu de transit provisoire, se transforme en un provisoire durable. Une telle zone grise dessine une situation analogue, en terme de suspension du temps dans un sas, à l'assignation au camp, durant la route migratoire ou déjà aux portes de l'Europe, en tant que forme " d'internement du présent " (Rahola, 2007) notamment pour des migrants ou demandeurs d'asile 
tout comme pour des aventuriers subsahariens dans un pays de transit (Pian, 2009).

L'allocation de droits scolaires est directement associée ici à une sorte de " différentiel allophonique " au sein du dispositif : ce différentiel, loin d'être homogène, place ces " allophones " dans une situation d'attentes multiples. Pour nos interlocuteurs "UPE2A italiens ", il s'agit d'un temps d'attente (au double sens de l'anglais waiting et expectation) qui concerne à la fois l'intégration définitive dans la classe ordinaire et leur façon de grandir en tant qu'adolescents pendant la fréquentation du dispositif. Ainsi, leurs processus d'autonomisation se mettent en place à l'intersection de plusieurs temporalités (Solberg, 1997) vécues dans des espaces dont le franchissement est bien contrôlé, à I'instar de I'UPE2A.

Une telle condition rappelle la manière dont, dans un traité de géographie humaine, Ratzel (2017) a introduit la notion de " bande de contention " (Grenzsaum, littéralement : le bord ou la bande de la frontière), au sens d'une zone grise où se superposent différentes formes de frontières, jusqu'à former un archétype de la frontière moderne représentant la frontière mieux que n'importe quelle abstraction linéaire. L'idée d'une bande de contention, dont l'épaisseur et la densité en indiquent la polysémie complexe, peut être tout particulièrement étendue à la situation liminale de ces "UPE2A italiens". Comme pour l'analyse des frontières contemporaines, où à côté du durcissement de la matrice linéaire et matérielle (les murs) on peut observer une véritable crise du concept de souveraineté étatique (Brown, 2010), ici s'impose un phénomène complémentaire. À l'instar des travaux montrant que les frontières contemporaines ont tendance à se manifester dans un ensemble incohérent de minuscules pratiques de contrôle et de surveillance (Bigo et Guild, 2003), avec des formes segmentées de citoyenneté (Balibar, 2015), sous la forme de points éparpillés (Cuttitta, 2007), I'allophonie tempérée des "UPE2A italiens " nécessite d'apprendre à jongler entre I'inclusion et l'exclusion, entre acceptation et résistance au dispositif. Or, cette condition est analogue à celle que doivent incorporer maints migrants, demandeurs d'asile et déboutés adultes, qui se trouvent constamment dans un seuil entre un " dedans " et un " dehors" (Khosravi, 2010).

Malgré ces fortes contraintes, il importe de souligner des conduites de vie et des actions à travers lesquelles ces adolescents négocient des initiatives de liberté auprès de leurs parents. En se retrouvant entre eux, souvent dans les centres commerciaux, ces élèves "UPE2A italiens " ont recours à la langue italienne bien que parfois ils le fassent à l'aide d'expressions en arabe. Ils manifestent la nostalgie du passé en Italie, y compris en dehors de l'école, pour tout ce qui a trait aux liens sociaux et aux relations d'amitié : ils sont au courant des évolutions du championnat italien de football comme d'autres évènements marquants sur le plan politique et culturel. Par ailleurs, ils consomment des " produits culturels " italiens. Cela inclut notamment la musique, à l'instar des chansons du chanteur rap Ghali : celui-ci est aimé par les adolescents italiens, mais il évoque aussi les contraintes parentales qui pèsent sur les enfants des 
Marocains ayant migré en Italie ${ }^{33}$.

\section{Conclusion : la frontière comme posture de recherche}

L'apport de notre contribution consiste à penser la reconfiguration contemporaine des frontières internes à I'Union européenne à partir d'un objet précis, tel que I' " allophonie " des "UPE2A italiens " de Strasbourg. Notre propos a été celui de déconstruire l'allophonie comme une forme contemporaine de matérialisation de l'espace représenté. L'allophonie se cache derrière sa dimension prétendument objective : le rapport au français scolaire des jeunes migrants. Si l'allophonie se met aujourd'hui en place à partir de critères légitimes pour le système éducatif français, ces critères ne sont ni seulement, ni tout à fait linguistiques et, dans le cas de I' " allophonie tempérée ", I'on voit bien que les élèves dits italiens puisqu'italophones sont renvoyés à une altérité sonore dépourvue de voix. II s'agit d'une altérité qui ne se cache pas de leur statut de migrants, sans pour autant pouvoir prendre en compte la condition de ces élèves ayant vécu une migration transalpine à Strasbourg, après être nés et avoir été scolarisés en Italie.

La condition de ces jeunes migrants renvoie à la hiérarchisation des statuts migratoires touchant également les mineurs, mais également à la relative facilité d'intégration que sont censés rencontrer des groupes plutôt que d'autres. C'est pourquoi nous avons abordé les modalités d'inclusion au collège par un rite d'institution, puis leur quotidien ordinaire caractérisé par une " inclusion différentielle ". Les formes de stigmatisation, ethnicisation et racisation étudiées au sein de l'école française sont multiples aujourd'hui ${ }^{34}$, mais peu de travaux se sont intéressés aux modalités de catégorisations tempérées susceptibles de développer des zones-frontières, des bandes de contention pour des " allophones de l'intérieur".

Nous avons essayé de relire l'expérience scolaire spécifique de jeunes migrants "italo-maghrébins " de Strasbourg au prisme des processus de reconfiguration générale des frontières européennes internes et extérieures. À l'instar des plus récents acquis apportés par les border studies (De Genova et al., 2015), les frontières contemporaines fonctionnent selon des pratiques irrégulières de suspension temporelle et de fragmentation spatiale. Celles-ci ont un impact direct sur les sujets dans la production d'une constellation de statuts différents ainsi que dans de multiples processus de ségrégation, segmentation et hiérarchisation. En ce sens, la frontière constitue un outil précieux d'analyse permettant d'interpréter notre matériau ethnographique. En d'autres termes, la

33 Le refrain d'une de ses chansons, Habibi, est " ti stanno addosso quando bevi ", sous-entendu les parents migrants originaires du Maroc en Italie te surveillent quand tu bois de I'alcool, afin d'en limiter la consommation. Cf. https://www.youtube.com/ watch?v=fgt6luBwzz0

$34 \mathrm{Cf}$. à titre d'exemple le colloque Racisme et discrimination raciale, de l'école à I'université, Université Paris Diderot, 27 au 29/09/2018, I'introduction et une partie des conférences sont accessible sur Canal U : https://www.canal-u.tv/video/universite_de_ nice_sophia_antipolis/1_ouverture_du_colloque_racisme_et_discrimination_raciale_dè_I ecole_a_I_universite.47277 
frontière est une véritable méthode (Mezzadra et Neilson, 2013). Les frontières sont à concevoir en tant qu' " espace heuristique " (Sassen, 2007) et en tant que véritables marges, points d'observation mobiles et privilégiés à la fois, favorisant I'analyse des politiques d'exclusion et de citoyenneté aujourd'hui. Si les frontières deviennent des points d'observation cruciaux, leur centralité suggère également une idée d'espace dans lequel la marge se fait centre, se superpose et reconfigure le centre lui-même. C'est précisément de cette centralité dont il est question dans l'expérience scolaire marginale des jeunes " italo-maghrébins " que nous avons rencontrés dans des classes ségréguées d'une ville-frontière.

\section{Références bibliographiques}

Agamben Giorgio (2013) The Endless Crisis as an Instrument of Power: In Conversation with Giorgio Agamben, [online] last checked on 30/01/2019 URL: https://www.versobooks.com/blogs/1318-the-endless-crisis-as-an-instrument-ofpower-in-conversation-with-giorgio-agamben

Armagnague-Roucher Maïtena, Cossée Claire, Mendonça Catherine, Rigoni Isabelle et Tersigni Simona (2018) Étude sur la scolarisation des élèves allophones nouvellement arrivés (EANA) et des enfants issus de familles itinérantes et de voyageurs (EFIV), Rapport de recherche EVASCOL, Paris, Défenseur des Droits, $418 \mathrm{p}$.

Armagnague-Roucher Maïtena et Tersigni Simona (2019) Construction d'une catégorie d'action publique en matière éducative : l'émergence de l'" allophonie ", Émulations, 29, pp. 73-89.

Balbo Laura (1998) Racisme, antiracisme et nationalisme en Italie. La construction d'un scenario européen, in Andrea Rea Éd., Immigration et racisme en Europe, Bruxelles, Éditions Complexe, pp. 93-105.

Balibar Étienne (2015 [2012 in Italian]) Citizenship, Cambridge, Polity press, $180 \mathrm{p}$.

Barrère Anne (2013) La montée des dispositifs : un nouvel âge de I'organisation scolaire, Carrefours de l'éducation, 36 (2), pp. 95-116.

Barth Fredrik (1995 [1969]) Introduction, in Barth Fredrik Ed., Ethnic Groups and Boundaries. The Social Organization of Cultural Difference, Bergen-Oslo, Waveland press, Long Grove, $153 \mathrm{p}$.

Belondo Sandra (2016) Les locuteurs de langues de la migration en France : des répertoires plurilingues peu visibles, in Christine Hélot et Jurgen Erfurt Éds., L'éducation bilingue en France. Politiques linguistiques, modèles et pratiques, Limoges, Lambert-Lucas, pp. 442-457.

Bigo Didier, Bonelli Laurent et Thomas Deltombe (Éds.) (2008) Au nom du 11 septembre... Les démocraties à l'épreuve de l'antiterrorisme, Paris, La Découverte, $420 \mathrm{p}$.

Bigo Didier et Guild Elspeth (2003) La mise à l'écart des étrangers. La logique du Visa Schengen, Cultures \& Conflits, 49, 147 p.

Bourdieu Pierre (1986) Rites comme actes d'institution, in Pierre Centlivres et Jacques Hainard Éds., Les rites de passage aujourd'hui, Lausanne, L'âge de I'homme, pp. 206-2015. 
Bourdieu Pierre (1982) Les rites comme actes d'institution, Actes de la Recherche en Sciences Sociales, 43, pp. 58-63.

Brown Wendy (2010) Walled States, Waning Sovereignty, New York, Zone books, $184 \mathrm{p}$.

Cassin Barbara (2004) Langue, in Cassin Barbara Éd., Vocabulaire européen des philosophies, Paris, Seuil/Le Robert, pp. 675-676.

Conseil de l'Europe (2014) Intégration linguistique des migrants adultes : Rapport final sur la 3e enquête du Conseil de l'Europe, Unité des Politiques linguistiques, Strasbourg, Projet ILMA, [en ligne]. URL : https://www.coe.int/fr/ web/lang-migrants/surveys

Cossée Claire, Laada Emmanuelle et Rigoni Isabelle (Éds.) (2004) Faire figure d'étranger. Regards croisés sur la production de l'altérité, Paris, Armand Colin, $319 \mathrm{p}$.

Cuttitta Paolo (2007) Le monde-frontière. Le contrôle de l'immigration dans I'espace globalisé, Cultures \& Conflits, 68, pp. 61-84.

De Genova Nicholas, Mezzadra Sandro and John Pickles (Eds.) (2015) New keywords: migration and borders, Cultural studies, 29 (1), pp. 55-87.

Descombes Vincent (1996) L'esprit objectif, in Vincent Descombes, Les institutions du sens, Paris, Minuit, pp. 267-308.

Detienne Marcel (2003) Comment être autochtone. Du pur Athénien au Français raciné, Paris, Seuil, $174 \mathrm{p}$.

Foucault Michel (2004) Sécurité, territoire, population, Paris, Gallimard/Seuil, $448 \mathrm{p}$.

Foucault Michel (2001) Espace, savoir et pouvoir, in Michel Foucault, Dits et Écrits II, Paris, Gallimard, 1091 p.

Foucault Michel (1997) Il faut défendre la société. Cours au Collège de France (1976), Paris, Seuil/Gallimard, 304 p.

Foucault Michel (1994) Dits et Écrits, 1954-1988. Tome III : 1976-1979, Paris, Gallimard.

Giglioli llaria, Hawthorne Camilla and Alessandro Tiberio (Eds.) (2017) Rethinking "Europe" through an ethnography of its borderlands, peripheries and margins, Etnografia e ricerca qualitativa, 10 (3), pp. 335-452.

Gordon Milton (1964) Assimilation in American Life. The Role of Race, Religion, and National Origins, New York, Oxford University Press, 276 p.

Gourdeau Camille (2016) Un contrat au service de l'identité nationale, Plein Droit, 110 , pp. 32-35.

Guillaumin Colette (1972) L'idéologie raciste : genèse et langage actuel, La Haye, Mouton, $247 \mathrm{p}$.

Haber Stéphane (2008) Quelques remarques sur la critique de l'argent au début du livre I du Capital de Marx, Philosophique, 11, pp. 9-40.

Hachimi Alaoui Myriam (2014) Intégration et lien de citoyenneté. Le cas du Contrat d'Accueil et d'Intégration, in Serge Paugam Éd., L'intégration inégale. Force, fragilité et rupture des liens sociaux, Paris, PUF, pp. 429-444. 
Herberg William (1960 [1955 en anglais]) Protestants, catholiques et israélites : la religion dans la société aux États-Unis, Essai de sociologie religieuse, Paris, Éditions Spes, 286 p.

Khosravi Shahram (2010) "Illegal» traveller. An Auto-Ethnography of Borders, Basingstoke, Palgrave Macmillan, $160 \mathrm{p}$.

Lefebvre Henri (2000 [1974]) La production de I'espace, Paris, Anthropos, 485 p.

Lochak Danielle (2013) Intégrer ou exclure par la langue ?, Plein droit, 98 (3), pp. 3-6.

Lochak Danielle (1985) Étrangers : de quel droit ?, Paris, PUF, 256 p.

Macmillan Alexandre (2010) La biopolitique et le dressage des populations, Cultures \& Conflits, 78, pp. 39-53, [en ligne] consulté le 22/06/2018. URL : http:// journals.openedition.org/conflits/17959

Marie Claude-Valentin (1996) L'Union européenne face aux déplacements de populations. Logiques d'État face aux droits des personnes, Revue Européenne des Migrations Internationales, (12) 2, pp. 169-209.

Mezzadra Sandro and Brett Neilson (2013) Border as Method, or, the Multiplication of Labour, Durham/London, Duke University Press, 384 p.

Mezzadra Sandro and Brett Neilson (2012), Between Inclusion and Exclusion: On the Topology of Global Space and Borders, Theory, Culture \& Society, 29 (4-5), pp. 58-75.

Miranda Adelina (2012) Les enfants d'immigrés en Italie entre assignations et revendications, Migrations Société, 141-142 (3), pp. 59-70.

Muni Toké Valelia (2009) Fantasmes d'un plurilinguisme pathogène : le cas des rapports dits "Bénisti ", Le français aujourd'hui, 1 (164), pp. 35-44.

New Keywords Collective (2016) Europe/Crisis: New Keywords of "the Crisis" in and of "Europe", Near Futures Online, 1 (1), pp. 1-45, [online] last checked on 20/11/2018. URL: http://nearfuturesonline.org/europecrisis-new-keywords-of-crisis-in-and-of-europe/

Olivier De Sardan Jean-Pierre (2008) La rigueur du qualitatif. Les contraintes empiriques de l'interprétation socio-anthropologique, Louvain La Neuve, Academia Bruylant, 368 p.

Palidda Salvatore (2011) Migrations critiques : Repenser les migrations comme mobilités humaines en Méditerranée, Paris, Éditions Karthala. 420 p.

Pian Anaïk (2009) Aux nouvelles frontières de l'Europe, L'aventure incertaine des Sénégalais au Maroc, Paris, La Dispute, 237 p.

Pian Anaïk, Hoyez Anne-Cécile et Tersigni Simona (2018) L'interprétariat en santé mentale : divisions sociale, morale et spatiale du travail dans les soins aux migrants, Revue Européenne des Migrations Internationales, 34 (2\&3), pp. 55-78.

Rahola Federico (2007) La forme-camp. Pour une généalogie des lieux de transit et d'internement du présent, Cultures \& Conflits, 68, pp. 31-50, [en ligne]. URL : http://conflits.revues.org/5213

Ratzel Friedrich (2017 [1882-1891]) Anthropogeographie: Die geographische Verbreitung des Menschen, Berlin, Verlag Der Wissenschaften, 640 p. 
Revel Judith (2013) Biopolitique et bio-pouvoirs, Implications philosophiques, [en ligne]. URL : http://www.implications-philosophiques.org/actualite/une/ biopolitique-et-biopouvoirs/

Rigouste Mathieu (2007) L'ennemi intérieur, de la guerre coloniale au contrôle sécuritaire, Cultures \& Conflits, 67, pp. 157-174, [en ligne]. URL : http://conflits. revues.org/3128

Sassen Saskia (2007) A Sociology of Globalization, New York, W.W. Norton \& Company, 250 p.

Sayad Abdelmalek (1999) Immigration et "pensée d'État ", Actes de la recherche en sciences sociales, 129 , pp. 5-14

Solberg Anne (1997) Negociating Childhood: Changing Constructions of Age for Norwegian Children, in James Alllison and Alan Prout Eds., Constructing and Reconstructing Childhood, London/New York, Routledge, pp. 126-144.

Tazzioli Martina and Garelli Glenda (2018) Containment beyond detention: The hotspot system and disrupted migration movements across Europe, Environment and Planning D: Society and Space, pp. 1-19.

Trucco Daniela (2015) Jeunes, musulmans et italiens (ou presque). Représentations de la citoyenneté et rapport au politique d'un groupe d'enfants d'immigrés à Gênes, Pôle Sud, 2 (43), pp. 29-42.

Van Gennep Arnold (1909) Les Rites de passage : études systématiques des rites, Paris, Librairie Émile Nourry, 288 p.

Virno Paolo (2001) Grammatica della moltitudine: per una analisi delle forme di vita contemporanee, Catanzaro, Rubbettino, 86 p. 


\section{Simona Tersigni et Lorenzo Navone}

\section{La zone grise de I'allophonie : " mouvements secondaires " et scolarisation des "UPE2A italiens " de Strasbourg}

Le recours à la catégorisation institutionnelle d'élèves allophones mérite d'être éclairé par l'analyse de la singularité dans la prise en charge de ceux qui bénéficient du dispositif UPE2A (Unité pédagogique pour les élèves allophones arrivants) en provenance d'Italie et d'ascendance nord-africaine. Mené à Strasbourg, notre terrain d'enquête montre qu'après un début de scolarisation en Italie depuis la maternelle, ces jeunes s'établissent en France avec leur famille. Leur allophonie est scolairement jugée peu problématique par I'institution et pourtant sa dimension atténuée d'altérité linguistique, mais aussi sociale et culturelle questionne. Les modalités d'accueil et d'accessibilité de ces élèves aux dispositifs spécialisés et à la classe ordinaire dessinent une bande de contention à l'intérieur d'une zone de relégation qui se justifie par leurs besoins spécifiques en français de scolarisation. Ce cas d'étude spécifique montre que l'école publique crée ses propres frontières à l'intérieur d'une dialectique de captation à l'égard de la relocalisation en cours des frontières européennes. Ces enfants et adolescents arrivant dans le cadre des " mouvements secondaires " prouvent l'autonomie de leur famille face à la crise économique et celle de l'accueil des nouveaux pays d'immigration de l'Europe du Sud. Ils mettent également à l'épreuve l'institution scolaire française, en dévoilant des politiques éducatives d'urgence qui risquent à long terme de réinjecter les effets de leur segmentation au-delà de l'espace scolaire. Enfin, I'“ allophonie tempérée " des élèves italo-nord-africains permet de repenser la redéfinition des frontières internes à l'Europe politique. Fonctionnant selon des pratiques irrégulières de suspension temporelle et de fragmentation spatiale, ces frontières exercent un impact sur les sujets dans la production d'une constellation de statuts différents ainsi que dans de multiples processus de classements et ségrégation.

\section{The Gay Zone of Allophony: "Secondary Mmovements" and Schooling of the "Italians UPE2A" in Strasbourg}

The institutional category of "allophone [non French-speaking] student" is here questioned by the analysis of a specific case study: namely, students of North African origin arriving from Italy and entering the UPE2A (pedagogical unit for allophone incoming students) in Strasbourg. After having been educated in Italy since preschool, such students migrated to France along with their families as a result of a "secondary movement". Since they are Italian speakers, their allophony is considered as "less problematic" by the school system, and yet the attenuate dimensions of their linguistic, cultural and social otherness is investigated. The students' access to both specific and ordinary classes draws a "containment strip" into a relegation zone, which is justified by their specific needs in learning French. In the context of the ongoing reconfiguration of European borders, this specific case study shows how the public school system creates its own boundaries. These children and teenagers, arriving in the context of "secondary movements" within Europe, show their families' autonomy from the economic and reception crisis hitting Southern European countries. Their presence seems to challenge the French education system: while unveiling emergency education policies, their segmentation risks, in the long 
run, to extend its effects beyond the school space. The "mitigated allophony" of the Italian/North African students allows to consider the reconfiguration of the internal borders of political Europe. Such boundaries function accordingly to irregular practices of temporal suspension and spatial fragmentation, and thus have an impact on the subjects involved. In practice, these boundaries produce a constellation of different statuses as well as processes of multiple classification and segregation.

\section{La zona gris de la alofonía: «movimientos secundarios» y escolarización de los "UPE2A italianos" de Estrasburgo}

La categorización institucional de "estudiantes alófonos [no francófonos]" se ilustra aquí a través un estudio de caso específico: a saber, el de los estudiantes originarios del norte de África, procedentes de Italia, que se benefician del dispositivo UPE2A (unidad pedagógica para estudiantes alófonos recién llegados) en las escuelas de Estrasburgo. Estos estudiantes, habiendo recibido educación en Italia desde el parvulario, emigraron a Francia junto con sus familias. Por el hecho de hablar italiano, su alofonía es considerada "menos problemática" que otras en el sistema escolar, en la cual se investiga la dimensión atenuada de su alteridad lingüística, cultural y social. Los criterios de acceso de estos estudiantes a los dispositivos especializados y a las clases ordinarias muestran cómo quedan dibujan una banda de restricción dentro de una zona de confinamiento que se justifica por las necesidades específicas del aprendizaje del francés. Este estudio de caso muestra que la escuela pública acaba creando sus propias fronteras especularmente a la reubicación de las fronteras europeas. Estos niños y adolescentes, que llegan en el marco de los «movimientos secundarios", muestran una autonomía de su familia con respecto a la crisis económica que ha afectado los nuevos países de inmigración del sur de Europa. Los estudiantes alófonos también cuestionan las instituciones educativas francesas, y revelan como las políticas de emergencia en el marco educativo a largo plazo comportan el riesgo de extender los efectos de esta segmentación más allá del espacio escolar. Finalmente, la "alofonía mitigada" de los estudiantes italianos/ norteafricanos permite considerar la redefinición de las fronteras internas de la Europa política. A medida que se extienden las prácticas irregulares de suspensión temporal y fragmentación espacial, estos límites marcan a estos sujetos a través de la producción de una constelación de diferentes status, así como de múltiples procesos de clasificación y segregación. 